Chapter 4

\title{
Neoadjuvant Chemotherapy: Role in Breast Cancer
}

\author{
Jasmeet Chadha Singh and Amy Tiersten \\ Additional information is available at the end of the chapter \\ http://dx.doi.org/10.5772/53192
}

\section{Introduction}

Neoadjuvant chemotherapy (NAC), also referred to as preoperative or primary chemotherapy refers to chemotherapy administered prior to tumor resection. It is a standard of care for management of locally advanced or inoperable breast tumors.

\section{Rationale for NAC}

The clinical rationale of NAC administration lies in the fact that it significantly downstages the existing tumor, enabling greater breast conservation (BSC). Although preoperative chemotherapy has not been shown to improve disease free (DFS) and overall survival (OS) for breast cancer when compared to post-operative therapy in operable patients, achievement of pathological complete response (pCR) defined as absence of any residual invasive tumor, is an important predictor of superior DFS and OS. In the B-27 trial looking at addition of taxanes to anthracycline based regimen in NAC, after 8 years of follow, patients who achieved pCR had superior DFS (HR: 0.49, p<0.0001) and OS (HR: 0.36, P<0.0001) rates. [1]- [5] The more recent I-SPY 1 study amongst several other studies [6]- [8], have found pCR to be an important predictor of recurrence free survival. [9], [10]

NSABP B-18 study was one of the earliest trials comparing neoadjuvant to adjuvant chemotherapy. The regimen of choice in this trial was the combination of adriamycin and cyclophosphamide (AC) either pre- or post operatively in 1523 women with operable palpable (T1-3, N0-1, M0) newly diagnosed breast cancer. There was no difference in overall survival between the two groups. However, a significantly greater number of patients underwent BCS in the NAC group (67vs. $60 \%$, p=0.002). 
In another trial of 1355 women with operable breast cancer, doxorubicin and paclitaxel followed by CMF in the neoadjuvant setting yielded similar RFS and OS rates when compared to adjuvant chemotherapy. However, patients with neoadjuvant chemotherapy achieved much higher breast conservation rates (63\% vs. $34 \%$, $\mathrm{p}<0.001)$. Distant relapse free survival was inferior in patients who did not achieve pCR (HR, 0.43; p- 0.025). [11]

\section{Prognostic factors}

Clinical trials have described various clinical and histological features of breast tumors, which may predict response to neoadjuvant chemotherapy. Higher nuclear grade is a significant predictor of pCR with NAC in several studies. [12]- [17] Proliferation index or Ki-67 [18] is shown to correlate positively to response. In the I SPY 1 trial, pCR rates in patients with high Ki-67 (defined as $>20$ percent) were $35 \%$ vs. $5 \%$ in patients with low Ki-67 (defined as $<10$ percent). [19] [9], [20] Pathologic examination of 82 breast cancer tumors after NAC with paclitaxel followed by 5 -fluorouracil, adriyamicin and cyclophosphamide ( $\mathrm{T} \cdot \mathrm{FAC}$ ) showed that basal like tumor pathology was a predictor of good response to NAC. [21] In the I SPY 1 trial luminal A histology had lowest pCR rates. [8], [19], [22] Negative estrogen and progesterone receptor status has shown to predict better response to NAC [7], [8], [13], [18], [23] In the I-SPY 1 trial pCR was highest for hormone receptor negative and HER-2 positive cancer (54\%) and lowest for HR+/ Her-2 negative cancer (9\%). [19], [24] In a study of 388 patients in which 16 percent patients were Her-2 positive, Her-2 positivity and young age were important predictors of achievement of PCR with anthracycline based NAC in univariate analysis. [25]

There is no single genetic marker that predicts complete response to NAC. However, gene expression profiling has been studied to predict response to various chemotherapy regimens with reasonable accuracy. [26] [27], [28] van't Veer et al developed a 70-gene expression model for prognostication using microarray analyses on 117 breast tumors. They found that genes associated with poor prognosis regulated cell cycle, metastasis, invasion and angiogenesis (eg. cyclin E2, MCM6, metalloproteinases MMP 9, MP1, RAB6B, PK428, VEGF receptor FLT1). [29] In the ISPY 1 trial, patients with p-53 null mutations and $17 \mathrm{q}$ amplification were also associated with high pCR rates ( $47 \%$ and $45 \%$ respectively). [19]

Enzymes of cytochrome P450 family play an important role in cancer drug metabolisms and polymorphism CYP2C9*2 polymorphism has been found to be associated with NAC resistance. [30] Tumor stage, lymph node positivity and dose intensity of chemotherapy have not been found to correlate to NAC response. [31] [17], [32]

Apoptotic responses to first dose of NAC measured by serial fine needle aspirations during first 4 days after chemotherapy administration was also found to be an indicator of response. [33]. Persistently elevated levels of CXCR4, a G-protein coupled receptor post chemotherapy has been found to be a negative predictor for response. [34]. A retrospective study of 562 patients concluded that metaplastic, mucinous and apocrine carcinoma responded poorly to NAC. [35] 


\section{Chemotherapy regimens}

In the pre-taxane era, initial trials of NAC were performed with anthracycline based regimens, such as AC [3]; 5-Fluorouracil, Adriyamicin and Cyclophosphamide (FAC) $)^{36}$ and 5-Fluorouracil, Epirubicin, Cyclophosphamide (FEC). [37] However, more recent trials have demonstrated that taxanes, when added to anthracycline based regimens, significantly improve survival outcomes [38] and therefore, should be used in combination with anthracycline based regimens. NSABP B-27 trial was designed to observe the impact of addition of 4 cycles of docetaxel to standard AC regimen in the preoperative setting. 2411 patients with T1C-3N0M0 or T1-3N1M0 breast tumors were assigned to ACX4 cycles vs. ACX 4 cycles followed by docetaxel X4 cycles vs. AC X 4 cycles preoperatively and docetaxel X 4 cycles postoperatively. Although the trial did not meet it's primary end point of demonstrating survival benefit, addition of docetaxel did double the pCR rates (from $13 \%$ to $26 \%$ ). pCR rates were also significant predictor of improved DFS (HR: 0.49) and OS (HR: 0.36) after more than eight years of follow-up. Patients who achieved clinical partial response with AC had significantly increased DFS with addition of docetaxel [39]

For patients with the Her-2 positive cancer, trastuzumab has been incorporated in the initial neoadjuvant chemoregimens. Options include sequential trastuzumab and paclitaxel and FEC in combination with trastuzumab ( $\mathrm{PH}-\mathrm{FECH})$ with $\mathrm{pCR}$ rates ranging from 55-65 percent. [40] However, in patients where cardiac morbidity is a concern docetaxel and cyclophosphamide in combination with trastuzumab (TCH) is also an effective choice. [41]

Capecitabine is an effective drug, which has yielded promising results in metastatic breast cancer. Capecitabine in combination of Vinorelbine has been found to be non inferior to Docetaxel, Adriyamicin, Cyclophosphamide (TAC) in terms of sonographic and pathologic complete response and breast conservation rates in the phase 3 Gepar trio trial [42]

\section{Dose dense NAC}

Dose intensity is achieved by increasing the drug dose delivered per cycle of chemotherapy by either increasing dose or decreasing inter-treatment interval. The PREPARE trial used dose dense $(\mathrm{dd})$ and dose intensified regimens of E+ P followed by CMF and compared it with standard dose $\mathrm{EC} \rightarrow \mathrm{T}$ regimen. Patients were treated with $\mathrm{E}(\mathrm{dd}) \rightarrow \mathrm{T}(250 \mathrm{mg} / \mathrm{m} 2)(\mathrm{dd}) \rightarrow \mathrm{CMF}$, each 2 weekly for 3 cycles with or without darbopoeitin versus standard E $(90 \mathrm{mg} / \mathrm{m} 2)$ and C followed by $\mathrm{P}(150 \mathrm{mg} / \mathrm{m} 2)$ for four cycles $(\mathrm{EC} \rightarrow \mathrm{T})$. The $\mathrm{pCR}$ rate was higher in the dose-dense, dose-intensified group (18.7\% vs. $13.2 \%$; $=0.04)$. Patients with non-inflammatory breast cancer had significantly improved disease free and overall survival from the dose dense regimens. [43]. A few other trials demonstrate similar increased in PCR rates with dose dense chemotherapy. [44] Currently, the value of dose dense chemotherapy in breast cancer is unclear amongst unselected patients.

In a phase 3 trial (SWOG 0012), standard 3 weekly AC regimen was compared to weekly doxorubicin and daily oral cyclophosphamide with GCSF support in the neoadjuvant 
setting for inflammatory and locally advanced breast cancer. $\mathrm{PCR}$ rates with the dose dense regimen were superior only in stage IIIB breast cancer and IBC. There was no difference in DFS and OS. [44]

\section{Assessment of response after NAC: Definition of pCR}

In most studies, $\mathrm{pCR}$ has been defined as absence of any residual invasive tumor in the pathologically examined tissue. Prior studies have indicated that there is no survival difference in patients with no residual tumor (in-situ or invasive) versus patients with residual in-situ (non- invasive tumor) cancer. [45] In a study performed at MD Anderson, 2302 patients treated with NAC showed similar disease free, overall and local recurrence free survival for patients who had in-situ cancer at the end of treatment when compared to patients who had no residual cancer. [46] However a recently published pooled analysis of 6,377 patients trials shows that DFS is better in patients with no residual tumor when compared to patients with residual in-situ tumor (HR:1.74, 95\% CI 1.28-2.36, $\mathrm{p}<0.001$ ). This study concluded that definition $\mathrm{pCR}$ should strictly be limited to absence of residual invasive or in-situ tumor either in the breast or lymph nodes [47].

\section{Estrogen or progesterone receptor positive cancer}

Although the use of endocrine therapy in the adjuvant setting is very common, it's use in the neoadjuvant setting is relatively recent. Neoadjuvant endocrine therapy has shown to cause tumor shrinkage [48] and reduce tumor proliferation as evidenced by decrease in Ki-67 and other markers of proliferation. [49] pCR is less commonly observed, therefore, response assessment in most of the trials involving endocine therapies is clinical (palpation and radiological techniques) as well as pathological assessment of proliferation markers. [50]

Comparisons have also been made between the 3 aromatase inhibitors exemestane, letrozole or anastrozole, in the neoadjuvant setting. 377 postmenopausal women with stage II/III ER positive breast cancer were treated with neoadjuvant exemestane $(25 \mathrm{mg} / \mathrm{d})$, letrozole $2.5 \mathrm{mg} / \mathrm{d}$ and anastrozole $1 \mathrm{mg} / \mathrm{d}$ for $16-18$ weeks. Clinical response rate, which was the primary end point of the trial, was $62.9 \%, 74.8 \%$ and $69.1 \%$ respectively. Breast conservation rates were comparable amongst the three groups. No difference was observed in terms of Ki-67 levels or changes in KI-67 expression among all the groups suggesting that they have biological equivalent effects. Overall, Luminal A tumors were likely to have a preoperative endocrine operative index (PEPI) score of zero before surgery when compared to luminal B tumors. [51]

Combination of neoadjuvant hormone and chemotherapy is also being investigated. In a phase 3 trial of 101 post menopausal women with locally advanced hormone receptor positive breast (stage T3, T4 and/ or N2 N3) cancer, neoadjuvant chemotherapy with FAC combined with letrozole $2.5 \mathrm{mg}$ daily produced superior $\mathrm{pCR}(25.5 \% \mathrm{VS} .10 .2 \%$, $\mathrm{p}=0.049)$ and clinical complete response rates $(27.6 \%$ VS. $10.2 \%, P=0.037)$ when compared to FAC alone. [52] 
It has been postulated that phosphotidyl kinase 3/ AKT/ mTOR pathway may be involved in endocrine resistance. For this reason, mTOR inhibitor like everolimus have been combined with hormone therapy in clinical trials. In a phase 2 trial comprising of 270 untreated patients with ER positive breast cancer, the control group was treated with letrozole $(2.5 \mathrm{mg} /$ day $)+$ placebo while the experimental treatment group was treated with letrozole plus everolimus (10mg/day). Patients treated with letrozole plus mTOR (mammalian target of Rapamycin) inhibitor had significantly improved clinical response rates, as well as response rates as assessed by ultrasound and mammography. Decrease in the proliferation index Ki-67 was significantly more marked with the combination treatment. Toxicities with the combination group were higher with $52.9 \%$ patients in the combination group having treatment stopped or delayed as a result of toxicities (only $7.6 \%$ in the placebo group). It was inferred that mTOR inhibitor can significantly increase the efficacy of hormone therapy. [53]

\section{HER-2 receptor positive cancer}

Trastuzumab or herceptin $(\mathrm{H})$, a monoclonal antibody against HER-2 neu receptor, is an integral part of neoadjuvant chemotherapy in HER-2 positive tumors. In a phase 3 trial, FEC + Trastuzumab followed by $\mathrm{P}+$ trastuzumab has shown significantly higher $\mathrm{pCR}$ rates when compared to $\mathrm{FEC} \rightarrow \mathrm{P}$ alone (66.7\% vs. 25\%). [54]

In the NeOAdjuvant herceptin (NOAH) trial, patients with HER-2 positive inflammatory or locally advanced breast cancer were treated with neoadjuvant chemotherapy alone $(\mathrm{A}+\mathrm{P} \rightarrow$ $\mathrm{P} \rightarrow \mathrm{CMF}$ ) or neoadjuvant chemotherapy combined with neoadjuvant $\mathrm{H}$ (added to the $\mathrm{CMF}$ part of neoadjuvant chemotherapy). Addition of $\mathrm{H}$ not only improved the rates of pathological response, ( $50 \%$ vs. $26 \%$; $\mathrm{p}=0.002$ ) but also the rates of event free survival, which was the primary end point of the study. More patients were able to undergo breast conservation surgery with the addition of $\mathrm{H}$ (35 vs. $13 \% \mathrm{p}=0.07)$. [55]

Trastuzumab has been compared to lapatinib (L), a tyrosine kinase inhibitor which is a dual inhibitor of EGFR and Her-2 receptors, in the neoadjuvant setting. In the Gepar Quinto trial, patients were treated with standard chemotherapy with four cycles of Epirubicin and cyclophosphamide followed by four cycles of docetaxel along with either $\mathrm{H}(6 \mathrm{mg} / \mathrm{kg}$ every 3 weeks) or L (1250mg daily) starting on the day 1 of the first EC cycle till the day 21 of the fourth cycle of docetaxel. pCR rates with $\mathrm{H}$ were significantly higher (30\%) when compared to L ( $30 \%$ vs. $22 \% ; \mathrm{p}=0.04)$. Overall difference in the clinical response and the number of breast conservation surgeries between the two groups was not significant. Edema and dyspnea were more common with trastuzumab while rash and diarrhea were more common with lapatinib. [56]

Neo-Altto trial was a randomized phase 3 trial comparing dual (trastuzumab/lapatinib combination) versus single Her-2 receptor blockade (trastuzumab or lapatinib alone) for HER-2 positive breast cancer, $>2 \mathrm{~cm}$ in diameter along with a taxane in the neoadjuvant setting. 154 patients received $1500 \mathrm{mg}$ of PO lapatinib, 149 received $4 \mathrm{mg}$ IV trastuzumab $(2 \mathrm{mg} / \mathrm{kg}$ subsequent doses) and 152 received the combination of trastuzumab with $1000 \mathrm{mg}$ PO lapatinib. pCR rates were significantly higher with dual blockade (51.3 percent; $95 \%$ C.I. 
43.1-59.5\%) when compared to single blockade with trastuzumab alone $(29.5 \%$; $95 \%$ C.I. 22.4-37.5). No significant difference in the pCR rates between trastuzumab and lapatinib groups was observed $(\mathrm{p}=0.34)$. Grade 3 diarrhea and elevation of liver enzymes were more common side effects in the lapatinib (23.4\%) and lapatinib plus trastuzumab group $(21.1 \%)$ when compared to trastuzumab only group ( $2 \%)$. The rate of breast conservation surgery in all the three groups was similar. pCR rate was higher in the ER negative tumors. [57]

In the preliminary results of the NSABP B-41 trial presented in American Society of Clinical Oncology's (ASCO) annual meeting in 2012, when $\mathrm{H}$ is substituted with $\mathrm{L}$, the responses pCR rates are found to be comparable. This trial comparing $\mathrm{AC} \rightarrow$ weekly paclitaxel $(\mathrm{WP})+\mathrm{H}$ vs. $\mathrm{AC} \rightarrow \mathrm{WP}+\mathrm{L}$ vs. $\mathrm{AC} \rightarrow \mathrm{WP}+\mathrm{H}+\mathrm{L}$; showed that $\mathrm{pCR}$ rates with $\mathrm{H}$ and $\mathrm{L}$ were comparable $(52.5 \%$ for $\mathrm{T}$ vs. $53.2 \%$ for $\mathrm{L}$ ). $\mathrm{pCR}$ rates with the combination of both $\mathrm{T}$ and $\mathrm{L}$ with NAC was slightly higher but the results were not statistically significant $(62 \% \mathrm{p}=0.075)$. [58]

Trastuzumab has been combined with another humanized monoclonal antibody against HER-2, Pertuzumab, which binds the dimerization site of HER-2 receptor and inhibits ligand dependent signaling. The phase 2 multicenter Neosphere trial compared combinations of $\mathrm{H}+$ $\mathrm{T}$ (group A), H+ Pertuzumab $+\mathrm{T}$ (group B), Pertuzumab $+\mathrm{H}$ (group C) and pertuzumab $+\mathrm{T}$ (group D) as neoadjuvant treatment of Her-2 positive breast cancer. The $\mathrm{pCR}$ rates in group $\mathrm{B}$ was significantly higher $(45.8 \%, \mathrm{p}=0.0141)$ when compared to groups A, C or D $(29 \%, 16.8 \%$ and $24 \%$ respectively). Clinical responses to NAC were also highest in group B. The rate of febrile neutropenia was similar in the trastuzumab+ pertuzumab + chemotherapy group to the $\mathrm{H}+\mathrm{T}$ group and was 7-8\%. [59]

Trastuzumab combined with bevacizumab and chemotherapy was found to be very effective in Her-2 positive inflammatory breast cancer in the phase 2 BEVERLY- 2 trial. In this study, 52 patients were treated with FEC + Bevacizumab (cycles 1-4) and docetaxel +bevacizumab and trastuzumab (cycles 5-8). pCR was seen in 33 patients (63.5\%). The frequency of grade $3 / 4$ neutropenia was $48 \%$. [60]

\section{Role of bevacizumab}

Bevacizumab is a monoclonal antibody against vascular endothelial growth factor (VEGF), which has been found to be very effective when added to anthracycline- taxane based neoadjuvant therapy. In the phase 3 NSABP- 40 trial, [61] patients with T1c-T3/ N0-N2 were treated with the following regimens: Docetaxel $(\mathrm{T})$ alone followed by EC vs. $\mathrm{T}+$ Gemcitabine followed by EC vs. T + G + Bevacizumab followed by EC vs. T + Capecitabine (X) followed by $\mathrm{EC}$ vs. $\mathrm{T}+\mathrm{X}+$ Bevacizumab followed by EC. Addition of bevacizumab significantly increased the pCR rates in the breast, which was the primary end point of this study $(28.2 \%$ to $34.5 \%$, $\mathrm{p}=0.02$ ). $\mathrm{pCR}$ rate in breast and nodes (secondary end point) was also increased but the result was not significant. Rate of clinical complete responses was significantly increased with addition of bevacizumab. Effect of bevacizumab was more pronounced in hormone receptor positive tumor and higher tumor grade. Side effects observed with bevacizumab included 
significantly higher rates of hypertension, left ventricular systolic dysfunction, mucositis and hand-foot syndrome.

Another phase 3 trial (GEPAR QUITNO) consisting if 1948 HER-2 negative patients concluded that rates of $\mathrm{pCR}$ were significantly improved when bevacizumab was added to $\mathrm{EC} \rightarrow \mathrm{T}$ regimen (14.9\% with $\mathrm{EC} \rightarrow \mathrm{T}$ alone and $18.4 \%$ with $\mathrm{EC} \rightarrow \mathrm{T}+$ Bevacizumab). In this study, improvement in $\mathrm{pCR}$ was limited to patients with hormone receptor negative tumor (39 with bevicizumab VS. $28 \%$ without bevacizumab). Side effect profile of bevacizumab was similar to the abovementioned study. [62]

Findings of the above two studies have led to conflicting results. While former has shown benefit of bevacizumab in hormone receptor positive patients, the latter has shown benefit in hormone negative cancer. A phase 3 ARTemis trial is currently underway which compares addition of bevacizumab to standard chemotherapy to chemotherapy alone. The study is to finish recruitment in December 2012 and primary outcome analysis due by December 2013. [63]

However, in some other trials, addition of bevacizumab to chemotherapy has shown less efficacy with additional toxicity. In one study, 45 women with Her2 negative locally advanced breast cancer were treated with neoadjuvant AC + bevacizumab X4 cycles followed by TX+ bevacizumab X4 cycles, with pCR rates of only 9 percent with substantial added toxicity such as fatigue, mucositis and headache. [64]

Trials looking at pathological markers predicting response to bevacizumab have shown positive responses associated with negative hormone receptor status, high Ki67 index and changes in phosphorilation status of VEGF receptor 2 (

JClin Oncol30, 2012 (suppl; abstr 10595))

\section{NAC in triple negative breast cancer}

Triple negative breast cancer is a more aggressive form of breast cancer that has poor prognosis despite response to chemotherapy. TNBC has been found to be sensitive to platinum based treatment in the metastatic setting due to inherent genomic instability. Encouraged by success in the metastatic setting, trials have been conducted with platinum agents in the primary setting. Silver et al. treated 28 patients with stage II/III TNBC with four three weekly cycles of cisplatin. pCR was seen in 22\% patients; good pathological response (Miller Payne score of 3,4 or 5 ) in $50 \%$ and progression in $14 \%$ patients. Positive response to cisplatin in this study was associated with young age, low BRCA expression, BRCA-1 prominent methylation, p-53 frameshift or nonsense mutation and gene expression significant of E2F3 activation. [65] More pCR rates have been demonstrated in BRCA-1 mutated breast cancers with cisplatin than with conventional regimens such as AC or CMF. [66] In another study, 17 patients with triple negative breast cancers $>2 \mathrm{~cm}$ in size, were treated with weekly doxorubicin plus daily oral cyclphosphamide followed by weekly paclitaxel and carboplatin, 14 out of 15 assessable patients showed clinical response. pCR rate was $46.6 \%$. Seven patients had grade $3 / 4$ hematological toxicity with this combination. [67] Similar high pCR rates have been reported with 
neoadjuvant bevacizumab, docetaxel and Carboplatin combination. [68] However, in a multicenter phase 2 study, addition of carboplatin to standard $\mathrm{EC} \rightarrow \mathrm{T}$ regimen for basal like breast cancer (defined as ER-/PR-/Her2-/Cytokeratin 5/6+ and /or EGFR+) did not enhance efficacy of standard chemotherapy (pCR rates: $35 \%$ vs. $30 \%$ in Carboplatin vs. no Carboplatin group, $\mathrm{p}=0.6064)$. [69]

The phase 3 GeparQuinto study demonstrated that addition of Bevacizumab to conventional chemotherapy $(\mathrm{EC} \rightarrow \mathrm{T})$ can further improve pathological $\mathrm{CR}(\mathrm{pCR})$ rates in triple negative breast cancer. [62]. However, as mentioned above, these findings contradict with the NSABP B40 study where major benefit was obtained in hormone positive cancer. [70] Carboplatin in combination with weekly nab paclitaxel and bevacizumab is also currently being evaluated in a clinical study. [71]

Ixabepilone is a new class of semisynthetic microtubule inhibiting drugs which is derived from natural epithilones. It has shown promising results in metastatic and multidrug resistant (anthracyclines, capecitabine, taxanes) breast cancer. [72]- [76] In a phase 2 study designed to assess benefit of ixabepilone in the neoadjuvant setting, 161 patients were treated with four cycles of ixabepilone. pCR was observed in $18 \%$ in all patients, but in $29 \%$ of ER negative patients, ER gene expression was inversely related to response in this study. [77] A pooled analysis of data from five phase 2 and two phase 3 trials, pCR rates with ixabepilone in the neoadjuvant setting were $26 \%$ in TNBC vs. $15 \%$ in non TNBC. [77], [78] Newer non-taxane microtubule inhibiting agents such as eribulin are being evaluated as NAC for TNBC in clinical trials.

Poly ADP ribose polymerase (PARP) inhibitors inhibit the PARP-1 enzyme which is a DNA base excision repair enzyme and along with BRCA, is involved in cell's DNA repair. [79], [80] It's role in tumorigenesis in evidenced by it's upregulation seen in tumor cells, thus protecting cancer cell DNA from damage and cell death. [81] PARP inhibition leads to cell death by two mechanisms. First, it causes accumulation of single and double stranded DNA breaks causing subsequent cell death. Secondly, it causes sensitization to therapeutic DNA damage. [82] Two trials utilizing PARP inhibitor Iniparib in combination of preoperative setting are underway and results are expected soon.

\section{Role of neoadjuvant chemoradiation}

In a multi-institutional study, 105 patients with locally advanced breast cancer were treated with twice weekly paclitaxel $30 \mathrm{mg} / \mathrm{m} 2$ for $10-12$ weeks and radiation therapy (total $45 \mathrm{gy}$ ) over weeks 2-7. Trastuzumab was added to this regimen in patients found to be Her-2 positive. Pathological response (complete and partial) was achieved in 34\% patients and was found to be significantly higher in hormone receptor negative patients (54\%, $95 \%$ C.I. $36 \%-69 \%)$ and triple negative tumors (54\%). As expected, patients who achieved pathological response had higher disease free survival (57 months vs. not reached, HR: 2.85, $\mathrm{p}<0.001$ ) and overall survival (84 months vs. not reached, HR: 4.27). [83] 


\section{Monitoring response to NAC}

Assessment of radiological response especially when using MRI or PET scan is very useful since it may help in early differentiation of responders to NAC from non-responders.

Studies have shown that decrease in tumor volume and enhancement on contrast enhanced MRI is associated with major histopathological response. [84], [85] Loo et al showed that MRI was able to monitor response to NAC more accurately in TNBC and Her-2 positive subsets but not in ER+, Her-2 negative subsets. [86] The I-SPY-1 study found that decrease in tumor volume as assessed by MRI early during treatment with NAC was a better predictor of pathologic response than measurement of tumor diameter by physical examination alone.

PET CT is another valuable imaging modality for accurately predicting response to NAC early in the course if therapy. [87], [88] In a study of 33 patients treated with carboplatin based NAC, there was significant correlation between FDG PET metabolic response after first and third cycles and overall survival. [89]

Studies have compared MRI and PET scan as predictors of response to NAC. Choi et al found that compared to PET CT, MRI was highly predictive of pCR $(\mathrm{P}<0.005)$ and better than PET CT for monitoring response to NAC. [90] However, Rousseau et al found that using $60 \%$ cut off value for SUV, the sensitivity, specificity and negative predictive value of PET scan were $89 \%, 955$ and $85 \%$ respectively after two cycles of NAC. Values were much lower for US and mammography. Tateishi et al also found PET CT to be superior to DCE MRI for pCR prediction after 2 cycles of NAC. [91]

\section{Conclusion}

NAC is the standard of care in management of locally advanced and inoperable breast cancer. It significantly downstages the tumor, thereby permitting breast conservation surgery. Anthracycline and taxanes based regimens are most commonly used NAC regimens. For Her-2 positive tumors, trastuzumab should be included in the NAC regimen. Role of other targeted therapies in NAC is being investigated.

\section{Author details}

Jasmeet Chadha Singh and Amy Tiersten

New York University Medical Center, New York, NY, USA, USA 


\section{References}

[1] Chollet P, Amat S, Cure H, et al: Prognostic significance of a complete pathological response after induction chemotherapy in operable breast cancer. Br J Cancer 86:1041-6, 2002

[2] Rastogi P, Anderson SJ, Bear HD, et al: Preoperative chemotherapy: updates of National Surgical Adjuvant Breast and Bowel Project Protocols B-18 and B-27. J Clin Oncol 26:778-85, 2008

[3] Wolmark N, Wang J, Mamounas E, et al: Preoperative chemotherapy in patients with operable breast cancer: nine-year results from National Surgical Adjuvant Breast and Bowel Project B-18. J Natl Cancer Inst Monogr:96-102, 2001

[4] Bear HD, Anderson S, Smith RE, et al: Sequential preoperative or postoperative docetaxel added to preoperative doxorubicin plus cyclophosphamide for operable breast cancer:National Surgical Adjuvant Breast and Bowel Project Protocol B-27. J Clin Oncol 24:2019-27, 2006

[5] Kuerer HM, Newman LA, Smith TL, et al: Clinical course of breast cancer patients with complete pathologic primary tumor and axillary lymph node response to doxorubicin-based neoadjuvant chemotherapy. J Clin Oncol 17:460-9, 1999

[6] Zhang GC, Qian XK, Guo ZB, et al: Pre-treatment hormonal receptor status and Ki67 index predict pathologic complete response to neoadjuvant trastuzumab/taxanes but not disease-free survival in HER2-positive breast cancer patients. Med Oncol, 2012

[7] Liedtke C, Mazouni C, Hess KR, et al: Response to neoadjuvant therapy and longterm survival in patients with triple-negative breast cancer. J Clin Oncol 26:1275-81, 2008

[8] Carey LA, Dees EC, Sawyer L, et al: The triple negative paradox: primary tumor chemosensitivity of breast cancer subtypes. Clin Cancer Res 13:2329-34, 2007

[9] Esserman LJ, Berry DA, Demichele A, et al: Pathologic Complete Response Predicts Recurrence-Free Survival More Effectively by Cancer Subset: Results From the I-SPY 1 TRIAL--CALGB 150007/150012, ACRIN 6657. J Clin Oncol, 2012

[10] Untch M, Fasching PA, Konecny GE, et al: Pathologic complete response after neoadjuvant chemotherapy plus trastuzumab predicts favorable survival in human epidermal growth factor receptor 2-overexpressing breast cancer: results from the TECHNO trial of the AGO and GBG study groups. J Clin Oncol 29:3351-7, 2011

[11] Gianni L, Baselga J, Eiermann W, et al: Phase III trial evaluating the addition of paclitaxel to doxorubicin followed by cyclophosphamide, methotrexate, and fluorouracil, as adjuvant or primary systemic therapy: European Cooperative Trial in Operable Breast Cancer. J Clin Oncol 27:2474-81, 2009 
[12] Andre F, Mazouni C, Liedtke C, et al: HER2 expression and efficacy of preoperative paclitaxel/FAC chemotherapy in breast cancer. Breast Cancer Res Treat 108:183-90, 2008

[13] Osako T, Horii R, Matsuura M, et al: High-grade breast cancers include both highly sensitive and highly resistant subsets to cytotoxic chemotherapy. J Cancer Res Clin Oncol 136:1431-8, 2010

[14] Fisher ER, Wang J, Bryant J, et al: Pathobiology of preoperative chemotherapy: findings from the National Surgical Adjuvant Breast and Bowel (NSABP) protocol B-18. Cancer 95:681-95, 2002

[15] Horii R, Akiyama F, Ito Y, et al: Histological features of breast cancer, highly sensitive to chemotherapy. Breast Cancer 14:393-400, 2007

[16] Rouzier R, Pusztai L, Delaloge S, et al: Nomograms to predict pathologic complete response and metastasis-free survival after preoperative chemotherapy for breast cancer. J Clin Oncol 23:8331-9, 2005

[17] Wang J, Buchholz TA, Middleton LP, et al: Assessment of histologic features and expression of biomarkers in predicting pathologic response to anthracycline-based neoadjuvant chemotherapy in patients with breast carcinoma. Cancer 94:3107-14, 2002

[18] Sanchez-Rovira P, Anton A, Barnadas A, et al: Classical markers like ER and ki-67, but also survivin and pERK, could be involved in the pathological response to gemcitabine, adriamycin and paclitaxel (GAT) in locally advanced breast cancer patients: results from the GEICAM/2002-01 phase II study. Clin Transl Oncol 14:430-6, 2012

[19] Esserman LJ, Berry DA, Cheang MC, et al: Chemotherapy response and recurrencefree survival in neoadjuvant breast cancer depends on biomarker profiles: results from the I-SPY 1 TRIAL (CALGB 150007/150012; ACRIN 6657). Breast Cancer Res Treat 132:1049-62, 2012

[20] al. Ae: Role of proliferation in response to neoadjuvant chemotherapy in GEICAM/ 2006-03 and GEICAM/2006-14 breast cancer patients. J Clin Oncol, 2012

[21] Rouzier R, Perou CM, Symmans WF, et al: Breast cancer molecular subtypes respond differently to preoperative chemotherapy. Clin Cancer Res 11:5678-85, 2005

[22] Li XR, Liu M, Zhang YJ, et al: CK5/6, EGFR, Ki-67, cyclin D1, and nm23-H1 protein expressions as predictors of pathological complete response to neoadjuvant chemotherapy in triple-negative breast cancer patients. Med Oncol 28 Suppl 1:S129-34, 2011

[23] Keam B, Im SA, Kim HJ, et al: Prognostic impact of clinicopathologic parameters in stage II/III breast cancer treated with neoadjuvant docetaxel and doxorubicin chemotherapy: paradoxical features of the triple negative breast cancer. BMC Cancer 7:203, 2007

[24] Li XR, Liu M, Zhang YJ, et al: ER, PgR, HER-2, Ki-67, topoisomerase IIalpha, and $\mathrm{nm} 23-\mathrm{H} 1$ proteins expression as predictors of pathological complete response to neo- 
adjuvant chemotherapy for locally advanced breast cancer. Med Oncol 28 Suppl 1:S48-54, 2011

[25] Keskin S, Muslumanoglu M, Saip P, et al: Clinical and pathological features of breast cancer associated with the pathological complete response to anthracycline-based neoadjuvant chemotherapy. Oncology 81:30-8, 2011

[26] Dejeux E, Ronneberg JA, Solvang H, et al: DNA methylation profiling in doxorubicin treated primary locally advanced breast tumours identifies novel genes associated with survival and treatment response. Mol Cancer 9:68, 2010

[27] Ayers M, Symmans WF, Stec J, et al: Gene expression profiles predict complete pathologic response to neoadjuvant paclitaxel and fluorouracil, doxorubicin, and cyclophosphamide chemotherapy in breast cancer. J Clin Oncol 22:2284-93, 2004

[28] Zembutsu H, Suzuki Y, Sasaki A, et al: Predicting response to docetaxel neoadjuvant chemotherapy for advanced breast cancers through genome-wide gene expression profiling. Int J Oncol 34:361-70, 2009

[29] van 't Veer LJ, Dai H, van de Vijver MJ, et al: Gene expression profiling predicts clinical outcome of breast cancer. Nature 415:530-6, 2002

[30] Seredina TA, Goreva OB, Talaban VO, et al: Association of cytochrome P450 genetic polymorphisms with neoadjuvant chemotherapy efficacy in breast cancer patients. BMC Med Genet 13:45, 2012

[31] Huober J, von Minckwitz G, Denkert C, et al: Effect of neoadjuvant anthracyclinetaxane-based chemotherapy in different biological breast cancer phenotypes: overall results from the GeparTrio study. Breast Cancer Res Treat 124:133-40, 2010

[32] Iwase S, Yamamoto D, Kuroda Y, et al: Phase II trial of preoperative chemotherapy for breast cancer: Japan Breast Cancer Research Network (JBCRN)-02 trial. Anticancer Res 31:1483-7, 2011

[33] Symmans WF, Volm MD, Shapiro RL, et al: Paclitaxel-induced apoptosis and mitotic arrest assessed by serial fine-needle aspiration: implications for early prediction of breast cancer response to neoadjuvant treatment. Clin Cancer Res 6:4610-7, 2000

[34] Hiller DJ, Li BD, Chu QD: CXCR4 as a predictive marker for locally advanced breast cancer post-neoadjuvant therapy. J Surg Res 166:14-8, 2011

[35] Nagao T, Kinoshita T, Hojo T, et al: The differences in the histological types of breast cancer and the response to neoadjuvant chemotherapy: The relationship between the outcome and the clinicopathological characteristics. Breast 21:289-95, 2012

[36] Hortobagyi GN, Ames FC, Buzdar AU, et al: Management of stage III primary breast cancer with primary chemotherapy, surgery, and radiation therapy. Cancer 62:2507-16, 1988 
[37] van der Hage JA, van de Velde CJ, Julien JP, et al: Preoperative chemotherapy in primary operable breast cancer: results from the European Organization for Research and Treatment of Cancer trial 10902. J Clin Oncol 19:4224-37, 2001

[38] Buzdar AU, Singletary SE, Theriault RL, et al: Prospective evaluation of paclitaxel versus combination chemotherapy with fluorouracil, doxorubicin, and cyclophosphamide as neoadjuvant therapy in patients with operable breast cancer. J Clin Oncol 17:3412-7, 1999

[39] Bear HD, Anderson S, Brown A, et al: The effect on tumor response of adding sequential preoperative docetaxel to preoperative doxorubicin and cyclophosphamide: preliminary results from National Surgical Adjuvant Breast and Bowel Project Protocol B-27. J Clin Oncol 21:4165-74, 2003

[40] Buzdar AU, Valero V, Ibrahim NK, et al: Neoadjuvant therapy with paclitaxel followed by 5-fluorouracil, epirubicin, and cyclophosphamide chemotherapy and concurrent trastuzumab in human epidermal growth factor receptor 2-positive operable breast cancer: an update of the initial randomized study population and data of additional patients treated with the same regimen. Clin Cancer Res 13:228-33, 2007

[41] Chang H: Clinical Advantages of Neoadjuvant Docetaxel (T) and Carboplatin (C) \pm Trastuzumab $(\mathrm{H})$ in Locally Advanced Breast Cancer (LABC). Cancer Research 69, 2009

[42] von Minckwitz G, Kummel S, Vogel P, et al: Neoadjuvant vinorelbine-capecitabine versus docetaxel-doxorubicin-cyclophosphamide in early nonresponsive breast cancer: phase III randomized GeparTrio trial. J Natl Cancer Inst 100:542-51, 2008

[43] Untch M, Fasching PA, Konecny GE, et al: PREPARE trial: a randomized phase III trial comparing preoperative, dose-dense, dose-intensified chemotherapy with epirubicin, paclitaxel and CMF versus a standard-dosed epirubicin/cyclophosphamide followed by paclitaxel +/- darbepoetin alfa in primary breast cancer--results at the time of surgery. Ann Oncol 22:1988-98, 2011

[44] Ellis GK, Barlow WE, Gralow JR, et al: Phase III comparison of standard doxorubicin and cyclophosphamide versus weekly doxorubicin and daily oral cyclophosphamide plus granulocyte colony-stimulating factor as neoadjuvant therapy for inflammatory and locally advanced breast cancer: SWOG 0012. J Clin Oncol 29:1014-21, 2011

[45] Jones RL, Lakhani SR, Ring AE, et al: Pathological complete response and residual DCIS following neoadjuvant chemotherapy for breast carcinoma. Br J Cancer 94:358-62, 2006

[46] Mazouni C, Peintinger F, Wan-Kau S, et al: Residual ductal carcinoma in situ in patients with complete eradication of invasive breast cancer after neoadjuvant chemotherapy does not adversely affect patient outcome. J Clin Oncol 25:2650-5, 2007 
[47] von Minckwitz G, Untch M, Blohmer JU, et al: Definition and impact of pathologic complete response on prognosis after neoadjuvant chemotherapy in various intrinsic breast cancer subtypes. J Clin Oncol 30:1796-804, 2012

[48] Dixon JM, Renshaw L, Bellamy C, et al: The effects of neoadjuvant anastrozole (Arimidex) on tumor volume in postmenopausal women with breast cancer: a randomized, double-blind, single-center study. Clin Cancer Res 6:2229-35, 2000

[49] Anderson TJ, Dixon JM, Stuart M, et al: Effect of neoadjuvant treatment with anastrozole on tumour histology in postmenopausal women with large operable breast cancer. Br J Cancer 87:334-8, 2002

[50] Dowsett M, Ebbs SR, Dixon JM, et al: Biomarker changes during neoadjuvant anastrozole, tamoxifen, or the combination: influence of hormonal status and HER-2 in breast cancer--a study from the IMPACT trialists. J Clin Oncol 23:2477-92, 2005

[51] Ellis MJ, Suman VJ, Hoog J, et al: Randomized phase II neoadjuvant comparison between letrozole, anastrozole, and exemestane for postmenopausal women with estrogen receptor-rich stage 2 to 3 breast cancer: clinical and biomarker outcomes and predictive value of the baseline PAM50-based intrinsic subtype--ACOSOG Z1031. J Clin Oncol 29:2342-9, 2011

[52] Mohammadianpanah M, Ashouri $Y$, Hoseini S, et al: The efficacy and safety of neoadjuvant chemotherapy +/- letrozole in postmenopausal women with locally advanced breast cancer: a randomized phase III clinical trial. Breast Cancer Res Treat 132:853-61, 2012

[53] Baselga J, Semiglazov V, van Dam P, et al: Phase II randomized study of neoadjuvant everolimus plus letrozole compared with placebo plus letrozole in patients with estrogen receptor-positive breast cancer. J Clin Oncol 27:2630-7, 2009

[54] Buzdar AU, Ibrahim NK, Francis D, et al: Significantly higher pathologic complete remission rate after neoadjuvant therapy with trastuzumab, paclitaxel, and epirubicin chemotherapy: results of a randomized trial in human epidermal growth factor receptor 2-positive operable breast cancer. J Clin Oncol 23:3676-85, 2005

[55] Semiglazov V, Eiermann W, Zambetti M, et al: Surgery following neoadjuvant therapy in patients with HER2-positive locally advanced or inflammatory breast cancer participating in the NeOAdjuvant Herceptin (NOAH) study. Eur J Surg Oncol 37:856-63, 2011

[56] Untch M, Loibl S, Bischoff J, et al: Lapatinib versus trastuzumab in combination with neoadjuvant anthracycline-taxane-based chemotherapy (GeparQuinto, GBG 44): a randomised phase 3 trial. Lancet Oncol 13:135-44, 2012

[57] Baselga J, Bradbury I, Eidtmann H, et al: Lapatinib with trastuzumab for HER2-positive early breast cancer (NeoALTTO): a randomised, open-label, multicentre, phase 3 trial. Lancet 379:633-40, 2012 
[58] al Re: Evaluation of lapatinib as a component of neoadjuvant therapy for HER2+ operable breast cancer: NSABP protocol B-41. J Clin Oncol, 2012

[59] Gianni L, Pienkowski T, Im YH, et al: Efficacy and safety of neoadjuvant pertuzumab and trastuzumab in women with locally advanced, inflammatory, or early HER2positive breast cancer (NeoSphere): a randomised multicentre, open-label, phase 2 trial. Lancet Oncol 13:25-32, 2012

[60] Pierga JY, Petit T, Delozier T, et al: Neoadjuvant bevacizumab, trastuzumab, and chemotherapy for primary inflammatory HER2-positive breast cancer (BEVERLY-2): an open-label, single-arm phase 2 study. Lancet Oncol 13:375-84, 2012

[61] Bear HD, Tang G, Rastogi P, et al: Bevacizumab added to neoadjuvant chemotherapy for breast cancer. N Engl J Med 366:310-20, 2012

[62] von Minckwitz G, Eidtmann H, Rezai M, et al: Neoadjuvant chemotherapy and bevacizumab for HER2-negative breast cancer. N Engl J Med 366:299-309, 2012

[63] al. Ee: ARTemis: Randomized trial with neoadjuvant chemotherapy for patients with early breast cancer. J Clin Oncol, 2012

[64] Rastogi P, Buyse ME, Swain SM, et al: Concurrent bevacizumab with a sequential regimen of doxorubicin and cyclophosphamide followed by docetaxel and capecitabine as neoadjuvant therapy for HER2- locally advanced breast cancer: a phase II trial of the NSABP Foundation Research Group. Clin Breast Cancer 11:228-34, 2011

[65] Silver DP, Richardson AL, Eklund AC, et al: Efficacy of neoadjuvant Cisplatin in triple-negative breast cancer. J Clin Oncol 28:1145-53, 2010

[66] Byrski T, Gronwald J, Huzarski T, et al: Pathologic complete response rates in young women with BRCA1-positive breast cancers after neoadjuvant chemotherapy. J Clin Oncol 28:375-9, 2010

[67] al. Te: Results of the East Carolina Breast Center phase II trial of neoadjuvant metronomic chemotherapy in triple-negative breast cancer (NCT00542191). J Clin Oncol, 2012

[68] al. Ke: A multicenter phase II neoadjuvant trial of bevacizumab combined with docetaxel plus carboplatin in the treatment of triple-negative breast cancer: Korean Cancer Study Group (KCSG-BR 0905, NCT 01208480). J Clin Oncol, 2012

[69] al. EAe: Chemotherapy (CT) with or without carboplatin as neoadjuvant treatment in patients with basal-like breast cancer: GEICAM 2006-03-A multicenter, randomized phase II study. J Clin Oncol 29, 2011

[70] al. Be: Phase II neoadjuvant trial with carboplatin and eribulin mesylate in patients with triple-negative breast cancer. J Clin Oncol, 2012 
[71] al. Se: Neoadjuvant bevacizumab with weekly nab-paclitaxel plus carboplatin followed by doxorubicin plus cyclophosphamide (AC) for triple-negative breast cancer. J Clin Oncol, 2012

[72] Roche H, Yelle L, Cognetti F, et al: Phase II clinical trial of ixabepilone (BMS-247550), an epothilone B analog, as first-line therapy in patients with metastatic breast cancer previously treated with anthracycline chemotherapy. J Clin Oncol 25:3415-20, 2007

[73] Low JA, Wedam SB, Lee JJ, et al: Phase II clinical trial of ixabepilone (BMS-247550), an epothilone B analog, in metastatic and locally advanced breast cancer. J Clin Oncol 23:2726-34, 2005

[74] Perez EA, Lerzo G, Pivot X, et al: Efficacy and safety of ixabepilone (BMS-247550) in a phase II study of patients with advanced breast cancer resistant to an anthracycline, a taxane, and capecitabine. J Clin Oncol 25:3407-14, 2007

[75] Thomas E, Tabernero J, Fornier M, et al: Phase II clinical trial of ixabepilone (BMS-247550), an epothilone B analog, in patients with taxane-resistant metastatic breast cancer. J Clin Oncol 25:3399-406, 2007

[76] Thomas ES, Gomez HL, Li RK, et al: Ixabepilone plus capecitabine for metastatic breast cancer progressing after anthracycline and taxane treatment. J Clin Oncol 25:5210-7, 2007

[77] Baselga J, Zambetti M, Llombart-Cussac A, et al: Phase II genomics study of ixabepilone as neoadjuvant treatment for breast cancer. J Clin Oncol 27:526-34, 2009

[78] Perez EA, Patel T, Moreno-Aspitia A: Efficacy of ixabepilone in ER/PR/HER2-negative (triple-negative) breast cancer. Breast Cancer Res Treat 121:261-71, 2010

[79] Hanahan D, Weinberg RA: Hallmarks of cancer: the next generation. Cell 144:646-74, 2011

[80] Harper JW, Elledge SJ: The DNA damage response: ten years after. Mol Cell 28:739-45, 2007

[81] Ossovskaya V, Koo IC, Kaldjian EP, et al: Upregulation of Poly (ADP-Ribose) Polymerase-1 (PARP1) in Triple-Negative Breast Cancer and Other Primary Human Tumor Types. Genes Cancer 1:812-21, 2010

[82] Farmer H, McCabe N, Lord CJ, et al: Targeting the DNA repair defect in BRCA mutant cells as a therapeutic strategy. Nature 434:917-21, 2005

[83] Adams S, Chakravarthy AB, Donach M, et al: Preoperative concurrent paclitaxel-radiation in locally advanced breast cancer: pathologic response correlates with fiveyear overall survival. Breast Cancer Res Treat 124:723-32, 2010

[84] Martincich L, Montemurro F, De Rosa G, et al: Monitoring response to primary chemotherapy in breast cancer using dynamic contrast-enhanced magnetic resonance imaging. Breast Cancer Res Treat 83:67-76, 2004 
[85] Hylton NM, Blume JD, Bernreuter WK, et al: Locally Advanced Breast Cancer: MR Imaging for Prediction of Response to Neoadjuvant Chemotherapy--Results from ACRIN 6657/I-SPY TRIAL. Radiology 263:663-672, 2012

[86] Loo CE, Straver ME, Rodenhuis S, et al: Magnetic resonance imaging response monitoring of breast cancer during neoadjuvant chemotherapy: relevance of breast cancer subtype. J Clin Oncol 29:660-6, 2011

[87] Inokuchi M, Furukawa H, Kayahara M, et al: [The role of / 18FDG PET/CT for the initial staging and therapy in primary breast cancer]. Gan To Kagaku Ryoho 36:2526-31, 2009

[88] Schelling M, Avril N, Nahrig J, et al: Positron emission tomography using [(18)F]Fluorodeoxyglucose for monitoring primary chemotherapy in breast cancer. J Clin Oncol 18:1689-95, 2000

[89] Avril N, Sassen S, Schmalfeldt B, et al: Prediction of response to neoadjuvant chemotherapy by sequential F-18-fluorodeoxyglucose positron emission tomography in patients with advanced-stage ovarian cancer. J Clin Oncol 23:7445-53, 2005

[90] Choi JH, Lim HI, Lee SK, et al: The role of PET CT to evaluate the response to neoadjuvant chemotherapy in advanced breast cancer: comparison with ultrasonography and magnetic resonance imaging. J Surg Oncol 102:392-7, 2010

[91] Tateishi U, Miyake M, Nagaoka T, et al: Neoadjuvant chemotherapy in breast cancer: prediction of pathologic response with PET/CT and dynamic contrast-enhanced MR imaging--prospective assessment. Radiology 263:53-63, 2012 
